\title{
Factors associated with changes in quality of life in patients undergoing allogeneic haematopoietic stem cell transplantation
}

\begin{abstract}
A. KISCH, RN, PHD CANDIDATe, Department of Haematology, Skåne University Hospital (SUS), Lund, and Faculty of Health and Society, Malmö University, Malmö, S. LENHOFF, MD, PHD, SENiOr Research ASsociate/Associate PROFEssor, Department of Haematology, Skåne University Hospital (SUS), Lund, S. ZDRAVKOVIC, PHD, SENIOR LeCturer, Faculty of Health and Society, Malmö University, Malmö, \& I. BOLMSJÖ, PHD, SENIOr ReSEARCH associate/Associate professor, Faculty of Health and Society, Malmö University, Malmö, Sweden
\end{abstract}

KISCH A., LENHOFF S., ZDRAVKOVIC S. \& BOLMSJÖ I. (2012) European Journal of Cancer Care Factors associated with changes in quality of life in patients undergoing allogeneic haematopoietic stem cell transplantation

It is well known that patients undergoing allogeneic haematopoietic stem cell transplantation (HSCT) experience changes in quality of life. We investigated factors associated with quality of life changes in adult HSCT patients. The Functional Assessment of Cancer Therapy - Bone Marrow Transplantation (FACT-BMT) scale, supplemented with the Functional Assessment of Chronic Illness Therapy - Spiritual Well-being (FACIT-Sp) subscale, was administered on three occasions, immediately before transplantation, 100 days and 12 months after transplantation. Analyses of nine selected factors were made where changes in quality of life were found. Seventy-five patients were included and 40 of these completed the study. Emotional well-being was found to improve between the baseline and 100 days, while all other dimensions deteriorated, including overall quality of life. Physical and social/family well-being deteriorated between the baseline and the 12-month follow-up, while emotional well-being improved. The main factors associated with deteriorating quality of life over time were found to be significant infections, female gender and transplantation with stem cells from a sibling donor. In our further studies we aim to focus on the relationships between patients and sibling donors in order to improve the care. Careful attention must be paid to continuous adequate information during the transplantation procedure.

Keywords: quality of life, FACT-BMT, FACIT-Sp, allogeneic haematopoietic stem cell transplantation, sibling donor.

\section{INTRODUCTION}

Allogeneic haematopoietic stem cell transplantation (HSCT) offers a potential cure for a variety of diseases, mainly haematological malignancies. However, there is a significant risk of acute complications and late side effects

Correspondence address: Annika Kisch, Department of Haematology, Skåne University Hospital (SUS), S-221 85 Lund, Sweden (e-mail: annika.kisch@mah.se).

Accepted 12 March 2012

DOI: $10.1111 / j .1365-2354.2012 .01354 . x$

European Journal of Cancer Care, 2012 that can negatively influence a patient's quality of life (QoL) (Lee et al. 2006; Pidala et al. 2009). QoL is multidimensional and proven difficult to define, and authors of QoL studies have often used their own definitions (Pidala et al. 2009). In World Health Organization's definition QoL has been defined as 'an individual's perception of their position in life in the context of the culture and the value system in which they live, and in relation to their goals, expectations, standards, and concerns' (WHOQOL Group 1993). This definition reflects the view that QoL refers to a subjective evaluation which is embedded in a cultural, social and environmental context. QoL has been 
described as a dynamic and multifaceted concept related to several dimensions of well-being: physical, emotional, functional and social (Cella et al. 1993). Allogeneic HSCT has also been found to have an impact on spiritual wellbeing (Ferrell et al. 1992; Andrykowski et al. 2005; Wong et al. 2010). Within the same individual QoL can fluctuate over time, because of development and environmental factors, reflecting the individual's assessment of his/her life at any one time relative to his/her previous state and prior experiences (Ferrell et al. 1992; Heinonen et al. 2001a).

Quality of life analyses after allogeneic HSCT are complex for many reasons. First, the number of patients undergoing HSCT is not very large at any centre. Second, due to treatment complications and relapses, a drop-off in patients during follow-up is inevitable. Third, the patient group is always heterogeneous in many respects, for example, regarding diagnosis, disease stage and how the transplantation is performed. Fourth, a large number of QoL questionnaires are existing and there is no consensus regarding which measure should be used (Kopp et al. 2000; Pidala et al. 2009). Anyhow, research in QoL of patients who are treated with allogeneic HSCT is important for many reasons. One strong reason is that research in QoL might help patients and medical staff in comparing the objective outcome of transplantation therapies against subjective QoL expectations and assessment (Kopp et al. 2000). This could represent a step forward in a meaningful involvement of patients in clinical decision making. Another goal of research in QoL is the development of patient-orientated rehabilitation, or recovery, programmes (Baumann et al. 2010; Pidala et al. 2010). Furthermore, studies have shown that psychological factors may have predictive value for survival following allogeneic HSCT, and psychosocial as well as QoL indices can be used as predictors of response to cancer treatment (Andrykowski et al. 1994; Hoodin et al. 2004).

Previous studies on QoL after allogeneic HSCT have been heterogeneous in their design, assessment instruments and results. Most studies have found that overall QoL deteriorates immediately after HSCT and stabilises or improves after 3 months (McQuellon et al. 1998; Bevans et al. 2006; Lee et al. 2006). Emotional well-being has been reported to be most impaired before and immediately after transplantation, but to improve quickly, at the time of hospital discharge and up to 3 months after transplantation (McQuellon et al. 1998; Bevans et al. 2006). However, there are conflicting data regarding changes in physical and social well-being (McQuellon et al. 1998; Bevans et al. 2006; Lee et al. 2006).
Earlier studies have identified factors associated with QoL concerns after allogeneic HSCT. Graft-versus-host disease (GvHD) is known to be a major complication after allogeneic HSCT and to influence QoL in long-term survivors (Chiodi et al. 2000; Pallua et al. 2010; Wong et al. 2010). Other factors that have been reported to be associated with QoL concerns are gender, age at transplant, marital status, primary diagnosis and presence of sequelae (Syrjala et al. 1993; Chiodi et al. 2000; Heinonen et al. 2001b; Pallua et al. 2010; Wong et al. 2010). Our previous experience (Kisch et al. 2008) and other authors (Christopher 2000; Wiener et al. 2008; van Walraven et al. 2010) have shown that donating stem cells to a relative, and receiving stem cells from a relative might be a complex issue. Based on previous studies and our clinical experience, the investigated factors in this study were chosen in advance, on the grounds that they may have a possible impact on QoL concerns after allogeneic HSCT.

In summary, QoL studies, including studies identifying factors associated with QoL, are important in identifying areas that can be improved in the provision of information, support and clinical care to patients undergoing allogeneic HSCT, including patient-orientated rehabilitation programmes. There is also a need for repeated QoL studies due to the steady pace of developments in the field of allogeneic HSCT, for example in patient and donor selection and the treatments used for conditioning and GvHD prophylaxis. Therefore, the aim of the present study was to identify factors associated with changes in QoL from a baseline immediately before allogeneic HSCT until 100 days and 12 months after the transplantation.

We here present the results of a prospective QoL study performed on adult patients undergoing and surviving allogeneic HSCT at least for approximately 100 days at a university hospital in Sweden.

\section{METHODS}

\section{Patients and data collection}

Between 1 May 2005 and 31 December 2008 we approached 79 consecutive patients admitted for allogeneic HSCT at our hospital. The inclusion criteria were age $\geq 18$ years and ability to speak and write Swedish. Initially, 75 patients agreed to participate. The patients were asked to complete the same questionnaire on three occasions. The first occasion was at admission to hospital (baseline), before the start of conditioning treatment. The second occasion was 100 days after transplantation and the third 12 months after transplantation, which means that data collection was finished in December 2009. Figure 1 shows the numbers of respondents on 


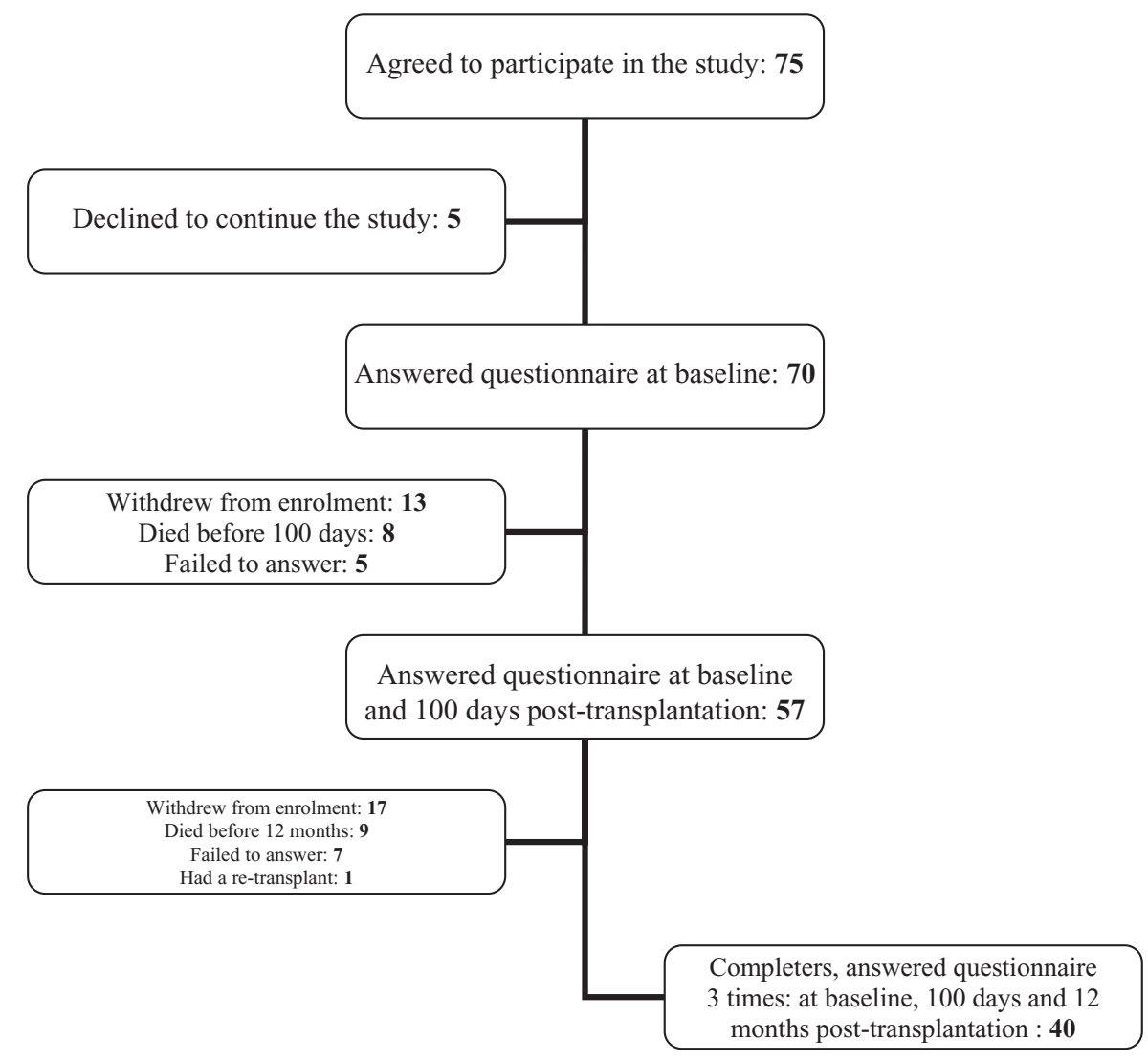

Figure 1. Flow chart of the patients from agreement to participate in the study until 12 months post-transplantation, participation and withdrawal. times: at baseline, 100 days and 12 months post-transplantation : 40 each occasion and the numbers of withdrawals. Seventy of the enrolled patients responded at admission (93\%), 57 patients also responded at 100 days $(76 \%)$ and 40 patients responded on all three occasions (completers) (53\%). The 57 patients who responded twice are included in the analysis of changes in QoL from baseline to 100 days after transplantation. The 40 completers are included in the analysis of changes in QoL from baseline to 12 months after transplantation and from 100 days to 12 months after transplantation. No attempts were made to assume answers on the QoL questionnaires for those patients being too ill or deceased, since the aim was to perform the study in patients surviving the transplantation for at least 100 days. Medical records were used to abstract clinical data about the patients. The study was approved by the regional ethical board of southern Sweden.

\section{Questionnaire}

The Functional Assessment of Cancer Therapy - Bone Marrow Transplantation scale (FACT-BMT), version 4 (Cella et al. 1993; McQuellon et al. 1997), was chosen for this study since it is a frequently used and recommended instrument in studies of QoL in patients undergoing allogeneic HSCT (Kopp et al. 2000). FACT-BMT gives a comprehensive overview of the multidimensional construct of QoL in patients undergoing HSCT with demonstrated reliability and validity (McQuellon et al. 1997; Kopp et al. 2000). To assess the patients' spiritual wellbeing, an additional subscale - Functional Assessment of Chronic Illness Therapy - Spiritual Well-being (FACITSp), version 4 (Peterman et al. 2002), was used in combination with FACT-BMT.

Functional Assessment of Cancer Therapy - Bone Marrow Transplantation is based on the 27-item cancerspecific questionnaire Functional Assessment of Cancer Therapy - General (FACT-G). FACT-G evaluates four dimensions of well-being using four subscales: physical well-being, social/family well-being, emotional wellbeing and functional well-being. The subscales deal with the following aspects: lack of energy, nausea and pain (physical well-being); sadness and worries about dying or getting worse (emotional well-being); working and enjoying life (functional well-being); and closeness to and support from friends and family (social/family well-being).

Functional Assessment of Cancer Therapy - Bone Marrow Transplantation includes an additional 23-item BMT subscale items specifically designed for patients who undergo HSCT/bone marrow transplantation (BMT). All 
23 items in this subscale were included in the questionnaire administered to the patients, but the scoring and analysis for the subscale is limited to 10 items in accordance with the FACIT guidelines (Cella 1997). In the development and validation manuscript for the BMT subscale, two of the 13 not included items have been set aside from the original version since they were not highly correlated with the remaining 10 items. Eleven of the 13 not included items were developed by an expert focus group of oncology/HSCT specialists in an effort to create a more comprehensive QoL instrument of HSCT concerns and have been added to the original scale but have not been through a psychometric evaluation (Cella 1997). FACTBMT provides an overall QoL score and has been validated on HSCT patients (McQuellon et al. 1997).

The FACIT-Sp consists of 12 items and is an optional subscale to the general scale (FACT-G) measuring spiritual well-being. FACIT-Sp is validated on people with cancer and other chronic illnesses (Peterman et al. 2002), and includes items dealing with sense of meaning and peace and the role of faith in illness, and it produces a total score for spiritual well-being.

Functional Assessment of Cancer Therapy - Bone Marrow Transplantation and FACIT-Sp are designed for self-administration, although they can also be administered in interview format. In this study the questionnaires were self-administered. In the questionnaires respondents are asked to use a 5-point Likert scale to rate how they have felt during the past 7 days with regard to each of a series of statements (items). Their answers are then scored according to scale-specific guidelines (Cella 1997). A higher score indicates better QoL (McQuellon et al. 1997; Peterman et al. 2002). Validated Swedish translations of the questionnaires were acquired from FACIT for use in the present study (Bonomi et al. 1996).

An overall QoL score was derived from the sum of the FACT-BMT and FACIT-Sp scores. Following the recommendations in the guidelines, the BMT-specific items were analysed both separately as a BMT subscale and in combination with the general form (FACT-G), as FACTBMT, in order to ensure high reliability (Cella et al. 1993; Cella 1997; McQuellon et al. 1997). Gaps in the completed baseline questionnaires were filled in consultation with the respondent. At 100 days and 12 months posttransplantation the questionnaires were mailed to the patients. No attempt was made to fill gaps in the completed questionnaires at 100 days and 12 months posttransplantation, based on the decision not to burden the patients more after completion of the questionnaires at home. Patients who did not answer the minimum number of items recommended by the FACIT guidelines at each of these stages were considered withdrawals and their responses excluded from the analysis.

\section{Factors analysed for association with changes in quality of life}

The factors included in the study were four baseline factors - age at transplant (20-49/50-65 years), gender, marital status at the time of transplantation (married or cohabiting/other than married) and disease status at transplant (standard risk/high risk) - and four treatmentspecific factors - donor (sibling donor/unrelated donor), conditioning regimen (myeloablative conditioning/ reduced intensity conditioning), GvHD 0-3 months post-transplantation (presence/absence) and significant infection 0-3 months post-transplantation (presence/ absence). Patients considered as 'other than married' were the patients who were single, separated, divorced, widowed or living apart. The number of patients who received transplants from a haploidentical donor (two patients) was considered too small to draw any conclusions; therefore, these patients were excluded in the analysis of the donor factor. Two additional factors included were GvHD 3-12 months post-transplantation (presence/ absence) and relapse 3-12 months post-transplantation (presence/absence). All relapses occurred more than 3 months post-transplantation.

\section{Definitions}

Standard-risk patients were defined as those with acute leukaemia in first remission, chronic myelogenous leukaemia in first chronic phase or aplastic anaemia without preceding immunosuppressive therapy. All other patients were considered high-risk.

Significant GvHD was defined as either need of corticosteroid therapy $>10 \mathrm{mg} /$ day for at least 14 days or organ impairment resulting in a loss of ordinary function.

Significant infection was defined as either need of treatment in inpatient care or need of treatment in outpatient care $\geq 3$ times a week.

\section{Statistical analysis}

Descriptive statistics were used to summarise demographic and clinical characteristics. Paired sample $t$-tests were used to test the significance of any changes in mean scores in the different dimensions of QoL over three periods: from baseline to 100 days post-transplantation, from baseline to 12 months post-transplantation and from 100 days to 12 months post-transplantation. Analyses to 
identify factors associated with significant changes in QoL scores were performed using one-way analysis of covariance (ANCOVA). In these analyses the calculated difference in QoL scores between compared time points was the dependent variable and the QoL baseline score the covariate. ANCOVA was further used by mutually adjusting for all significant factors. A $P$-value below 0.05 was considered statistically significant. The statistical analyses were performed using the Statistical Package for Social Sciences (SPSS ${ }^{\circledR}$ ) version 18.0 software (SPSS Inc., Chicago, IL, USA).

\section{RESULTS}

\section{Patient characteristics}

Demographics and clinical characteristics are shown in Table 1 for respondents at baseline, at 100 days posttransplantation $(n=57)$ and completers $(n=40)$. The mean age was 50 years in both groups. In the group of responders at baseline and at 100 days there were almost equal numbers of men and women (29 men, 28 women). In the group of completers there were 23 men and 17 women. There was a higher frequency of significant infection 0-3 months post-transplantation among those who responded at baseline and at 100 days than among the completers $(25 \%$ vs. $15 \%)$. The other characteristics were similarly distributed in the two groups.

\section{Quality of life from baseline to 100 days post-transplantation}

Changes in mean QoL scores from baseline to 100 days post-transplantation are presented in Table 2. Significant changes were found in all dimensions of QoL. Deteriorations were found in the majority of the dimensions of QoL: physical well-being $(P=0.000)$, social/family wellbeing $(P=0.003)$ and functional well-being $(P=0.009)$, the general scale (FACT-G) $(P=0.000)$, the subscale specific for HSCT patients (BMT) $(P=0.012)$, the BMT subscale in combination with the general scale (FACT-BMT) $(P=$ 0.000), the spiritual well-being (FACIT-Sp) $(P=0.043)$ and the overall QoL $(P=0.001)$, while emotional well-being improved from baseline to 100 days post-transplantation. The $P$-value for the change in mean scores for emotional well-being was 0.051 , which was considered as borderline significance. Factors associated with significant changes in QoL from baseline to 100 days post-transplantation are reported in Table 3. Significant infection 0-3 months posttransplantation (presence/absence) and gender (male/ female) were the two remaining significant factors after mutual adjustment. The presence of significant infection 0-3 months post-transplantation and female gender were associated with deteriorated QoL, while absence of significant infection was associated with improved emotional well-being.

\section{Quality of life from baseline to 12 months post-transplantation}

Changes in mean QoL scores for completers of the study are presented in Table 4. Significant changes from baseline to 12 months post-transplantation were found in physical, social/family and emotional well-being. Deteriorations were seen in physical well-being $(P=0.037)$ and in social/family well-being $(P=0.033)$ scores, while the emotional well-being score increased over the period $(P=$ 0.008). Factors associated with significant changes in QoL from baseline to 12 months post-transplantation are reported in Table 5. Three factors were associated with changes in QoL from baseline to 12 months posttransplantation: donor (sibling donor/unrelated donor), relapse 3-12 months post-transplantation (presence/ absence) and marital status (married or cohabiting/other than married). Patients transplanted with stem cells from a sibling donor showed deteriorated social/family wellbeing, the presence of relapse was associated with deteriorated physical well-being, while the marital status 'other than married' (i.e. single, separated, divorced, widowed or living apart) was associated with improved emotional well-being score.

There were no significant changes in QoL between 100 days and 12 months post-transplantation (Table 4).

\section{DISCUSSION}

The aim of this prospective single-centre study was to explore the association between a number of factors with changes in QoL over time in patients undergoing HSCT at our centre during a period of just over 3.5 years and surviving at least approximately 100 days. Significant deteriorations were found in the majority of the dimensions of QoL over time, including overall QoL, while emotional well-being improved over time. Overall QoL for the patients in this study deteriorated at 100 days posttransplantation and improved thereafter, which is in accordance with other studies (McQuellon et al. 1998; Bevans et al. 2006). The finding in this study regarding physical well-being at 100 days post-transplantation is also in accordance with other studies, where there is a consensus of a decline immediately after transplantation with a nadir at about 100 days (McQuellon et al. 1998; 
Table 1. Characteristics and clinical data of patients in the study

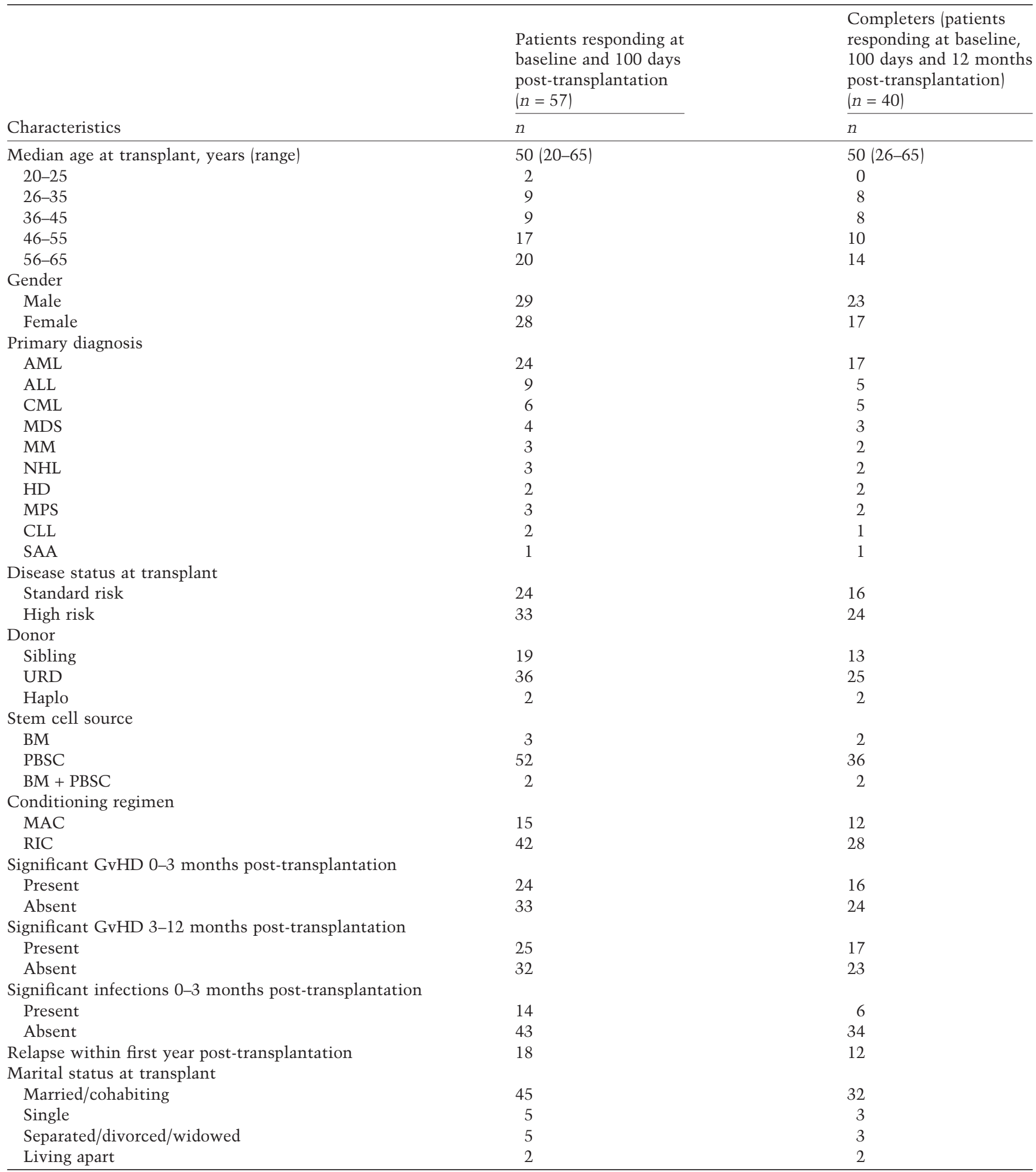

ALL, acute lymphatic leukaemia; AML, acute myeloid leukaemia; BM, bone marrow; CLL, chronic lymphatic leukaemia; CML, chronic myeloid leukaemia; GvHD, graft-versus-host disease; HD, Hodgkin's disease; MAC, myeloablative conditioning; MDS, myeloid dysplastic syndrome; MM, multiple myeloma; MPS, myeloproliferative syndrome; NHL, non-Hodgkin lymphoma; PBSC, peripheral blood stem cells; RIC, reduced intensive conditioning; SAA, severe aplastic anaemia; URD, unrelated donor. 
Table 4. Functional Assessment of Cancer Therapy - Bone Marrow Transplantation (FACT-BMT) and Functional Assessment of Chronic Illness Therapy - Spiritual Well-being (FACIT-Sp), mean scores at three time points and tests of significance for patients responding at baseline, 100 days post-transplantation and 12 months post-transplantation

\begin{tabular}{|c|c|c|c|c|c|c|}
\hline \multirow{3}{*}{$\begin{array}{l}\text { Dimension of quality of } \\
\text { life (range of possible } \\
\text { scores) }\end{array}$} & 1 & 2 & 3 & \multirow{3}{*}{$\frac{P \text {-value }}{1 \text { vs. } 2}$} & \multirow{3}{*}{$\frac{P \text {-value }}{1 \text { vs. } 3}$} & \multirow{3}{*}{$\frac{P \text {-value }}{2 \text { vs. } 3}$} \\
\hline & Baseline $(n=40)$ & 100 days $(n=40)$ & 12 months $(n=40)$ & & & \\
\hline & Mean (SD) & Mean (SD) & Mean (SD) & & & \\
\hline Social/family well-being (0-28) & $24.68(3.4)$ & $23.59(4.1)$ & $23.33(4.9)$ & 0.029 & 0.033 & 0.666 \\
\hline Emotional well-being $(0-24)$ & $17.20(4.9)$ & $18.93(4.2)$ & $19.16(5.4)$ & 0.021 & 0.008 & 0.726 \\
\hline Functional well-being (0-28) & $18.82(5.2)$ & $18.15(5.7)$ & $19.00(6.1)$ & 0.388 & 0.855 & 0.370 \\
\hline FACT-BMT (0-148) & $109.79(17.7)$ & $105.10(20.4)$ & $106.90(23.4)$ & 0.069 & 0.393 & 0.558 \\
\hline FACIT-Sp (0-48) & $35.95(7.6)$ & $35.56(8.0)$ & $35.80(7.5)$ & 0.631 & 0.883 & 0.494 \\
\hline Overall quality of life (0-196) & $145.51(23.1)$ & $140.66(26.1)$ & $143.96(28.5)$ & 0.125 & 0.603 & 0.222 \\
\hline
\end{tabular}

$P$-value $<0.05$ indicates a statistically significant difference.

FACT-G, Functional Assessment of Cancer Therapy - General scale; BMT, bone marrow transplantation/haematopoietic stem cell transplantation; FACT-BMT, Functional Assessment of Cancer Therapy - Bone Marrow Transplantation scale $=$ FACT-G + BMT subscale; FACIT-Sp, Functional Assessment of Chronic Illness Therapy - Spiritual Well-being subscale; Overall quality of life, FACT-BMT + FACIT-Sp.

Table 5. Factors associated with the significant changes in mean scores in the different dimensions of quality of life from baseline to 12 months post-transplantation, from ANCOVA analysis

\begin{tabular}{ll}
\hline $\begin{array}{l}\text { Dimension of quality of } \\
\text { life with significant } \\
\text { change in mean score }\end{array}$ & $\begin{array}{l}\text { Factors associated with } \\
\text { the change, one-way }\end{array}$ \\
\hline $\begin{array}{l}\text { Decreasing } \\
\text { Physical well-being }\end{array}$ & $\begin{array}{l}\text { Relapse } \\
\text { Social/family well-being }\end{array}$ \\
$\begin{array}{l}\text { Sibling donor } \\
\text { Emotional well-being }\end{array}$ & Other than married (marital status) \\
\hline
\end{tabular}

Other than married, marital status = single, separated, divorced, widowed or living apart.

ANCOVA, analysis of covariance.

for patients not married or cohabiting is that these individuals might experience less responsibility for others, compared to the ones who are married/cohabiting, which contributes to less worries, that is, worries to be a burden for others, especially the partner, which is also supported by Milne et al. (2006).

Emotional well-being scores increased over time, confirming the findings of earlier studies (McQuellon et al. 1998; Bevans et al. 2006). A likely explanation is that patients are anxious about dying before the transplantation and relieved at surviving the treatment. Emotional well-being was better for the group of completers compared to the whole group at 100 days post-transplantation. This is not surprising, as more seriously ill patients responded at 100 days than did at 12 months. This interpretation was supported by analysis of the individual items in the emotional well-being subscale, which showed those items dealing with feelings of nervousness, worries about dying and worries about getting worse improving considerably over time.

Analyses of the items in decreasing physical well-being and in the items specific for patients who had undergone HSCT/BMT confirmed fatigue as being a central problem (Danaher et al. 2006). The outstanding decreasing scores in these subscales were associated with lack of energy, physical condition as an obstacle to meeting the needs of the family, reduced appetite, reduced mobility and tiredness. Fatigue has in several studies been elucidated as a problem after HSCT. Almost one-third of the patients after HSCT in the study by Gruber et al. (2003) reported serious fitness problems. Fatigue is a well-known symptom related to HSCT (Edman et al. 2001; Larsen et al. 2007) and has been described as a multidimensional construct in nature with physical as well as psychological and social symptoms, with reduced physical fitness, extreme tiredness and weakness (van Weert et al. 2006). Andersson et al. (2011) showed that fatigue is a prominent problem during the first year after allogeneic HSCT, interfering with daily life. Social/family well-being scores in this study population decreased over time despite increases in emotional well-being. This apparent paradox may be partly explained by family and friends eventually losing the energy and strength to support the patient, even though the patient's support needs are still great. This explanation is supported by an analysis of the items in the social/family well-being subscale, which found those items concerned with emotional support from family and friends showing the most marked impairment over time. The family and friends of patients who undergo allogeneic HSCT are easily forgotten but probably need better 
support. This explanation supports the idea that patients' deteriorated social/family well-being and high levels of fatigue is well connected with the patients' deteriorated physical well-being. During the rehabilitation phase the caregivers must be aware of the importance of including the social context of the patient and the psychosocial adjustment as well as the physical functioning. This is also in accordance with what van Weert et al. (2006) have shown using a multidimensional rehabilitation programme on cancer-related fatigue, which effectively reduces patient-experienced fatigue and reaches an energy balance for the patients.

Presence of significant infections was the factor most clearly associated with decreasing overall QoL score, while the presence of GvHD was only associated with impaired physical well-being. Our interpretation of this result is that our patients are well informed about GvHD prior to transplantation, including the positive effects of GvHD on their disease. The patients are also informed about possible infections, but not to the same extent as they are about GvHD, and there is anyway nothing positive for the patient in having a severe infection. In addition, GvHD occurring 3-12 months post-transplantation was not a factor associated with any QoL concerns. This finding must, however, be interpreted with caution because the majority of the patients who did not answer the third questionnaire had died or were too ill mainly due to GvHD. Also, assessment of patients over a longer period would probably show a correlation between chronic GvHD and QoL, as has been found in other studies (Chiodi et al. 2000; Pallua et al. 2010; Wong et al. 2010), but this was not done since the purpose of this study was to investigate changes in QoL and associated factors in survivors up to 1 year after transplantation.

In this study, whether a patient received myeloablative or reduced conditioning had no impact on QoL. This could be because the reduced conditioning protocols used at our centre are relatively intensive and these patients usually experience mucositis, appetite loss and fatigue.

The association of female gender with impaired QoL accords with other studies indicating gender differences among patients undergoing allogeneic HSCT. In the study by Heinonen et al. (2001b) women were reported to have worse emotional well-being than men, and men to experience less satisfaction with social support than women. Prieto et al. (1996) reported that women had impaired overall QoL compared to men, and female survivors after HSCT had a higher rate of depression disorders than male survivors in the study by DeMarinis et al. (2009).
One finding we present is that patients transplanted with stem cells from a sibling donor experienced greater deterioration in social/family well-being than those given stem cells from an unrelated donor. We have previously reported that the identification of a possible sibling donor is not a straightforward issue (Kisch et al. 2008). The relationship between siblings can change considerably in this situation. The situation of sibling donors has been highlighted, by showing that sibling donors are in a vulnerable situation and experience fear, anxiety and ambivalence about the donation procedure (Fortanier et al. 2002; Williams et al. 2003; Bredeson et al. 2004; Wiener et al. 2008). However, also good experiences of pride for helping the very ill sibling have been reported by sibling donors (Christopher 2000). Sibling donors have also reported a need of more information, and they have expressed that their relationship with the ill sibling has changed in connection to the donation (Williams et al. 2003; Wiener et al. 2008). A sibling not able to donate, or not selected as a donor, reported feelings of alienation and emotional distress in the study by Takita et al. (2011), which also contributes to changes in relationships between the siblings, and in the QoL of the sibling donor and of the patient.

\section{Strengths and limitations}

One of the strengths of this study is that the study included all patients admitted for allogeneic HSCT at one centre (i.e. the same context) during a period of just over 3.5 years, where $95 \%$ (75 of 79 ) of the approached patients agreed to participate. Continuing inclusion of patients during a longer period of time would signify a risk of changes in treatment regimens with the result of a more heterogenic patient group in the study. Nevertheless, one limitation of this study might be its sample size as compared to multicentre studies. However, this is a total population study with a sample size comparable with other single-centre studies (Pidala et al. 2009). A second limitation might be that only FACT-BMT and FACIT-Sp were used for assessment of the patients' QoL, without combining with another measurement. McCabe et al. (2008) recommend the use of a combination of methods, such as questionnaires and Visual Analogue Scales or questionnaires and interviews for data collection in QoL studies. The use of mixed methods would provide greater meaning and understanding of the quantitative data and also identifying individual perspectives in relation to QoL issues. This facilitates interpretation of the difference between statistical and clinical significance of findings and for implication for patient care. However, FACT-BMT 
was chosen for this study since it is a recommended instrument specific to measurement of QoL in this population, giving a comprehensive overview of the multidimensional construct of QoL, with demonstrated reliability and validity (McQuellon et al. 1997). FACTBMT was combined with FACIT-Sp to assess the spiritual well-being, which has been found to be influenced by HSCT (Ferrell et al. 1992; Andrykowski et al. 2005; Wong et al. 2010). Thus, QoL data must always be interpreted carefully and comparisons between studies be made with caution.

\section{IMPLICATIONS FOR PRACTICE}

A number of complications can occur after allogeneic HSCT, influencing QoL. The patient should receive detailed yet balanced information before transplantation about the risks in order to be as mentally prepared as possible, also for unwanted complications such as infections. Continuous and repeated adequate information during the transplantation procedure and during the recovery period after transplantation is crucial for patients' recovery and QoL. One way to facilitate the patients' recovery period back to a normal life is, as Winterling et al. (2009) suggest, that health professionals inform and discuss expectations and plans for the recovery period together with the patient and the spouses when the patient is about to proceed to the recovery period. The information should include that the recovery period can be tough and often take a long time, and information about who they should turn to if they need help. The patients' status and how the recovery period is progressing should thereafter be evaluated at subsequent hospital appointments. At these appointments it will be possible to identify individual needs of professional help from different caregivers, such as physiotherapist, dietician, psychological support and the possibility to get answers to medical questions that turn up during the recovery period. Since many of the factors discussed are psychological and social in nature, interventions from a multidisciplinary team are required. Behavioural interventions including psychosocial interventions have been shown to result in improvements in fatigue (Carlson et al. 2006) and physical symptoms (Wilson et al. 2005) as well as improvements in overall QoL (Hayes et al. 2004; Baumann et al. 2010). As
Pidala et al. (2010) suggest, referral for these interventions is best achieved in a multidisciplinary team dedicated to the care of HSCT patients, which offers promise for improved QoL after transplantation.

Impairment of social/family well-being when transplanted with cells from a sibling donor compared to cells from an unrelated donor is an interesting finding. The reasons behind the impaired QoL when transplanted with cells from a sibling donor are somewhat unclear. Perhaps, the patient should be asked about the relationship with the sibling before the transplantation, and what feelings the patient has towards this sibling donor, and his or her role as a donor. After the transplantation, the patient's feelings again can be addressed, in order to identify negative emotions and give a professional support.

The situation with a sibling donor in allogeneic HSCT has been described in earlier studies, but future studies are needed to highlight and focus on the relationships between patients and sibling donors in order to improve the care of both the patients and siblings. An analysis of the experiences of siblings being asked to undergo human leukocyte antigen typing is ongoing, and an interview study with up to 10 recipient-sibling donor pairs in order to investigate their perception of each other's situation is also ongoing.

\section{ACKNOWLEDGEMENTS}

We would like to thank all the patients who willingly took part in this study and thanks to Azzam Khalaf for his help with the statistics.

\section{STATEMENT OF AUTHORSHIP}

Annika Kisch and Stig Lenhoff designed the study. Annika Kisch and Slobodan Zdravkovic conducted the analysis. Annika Kisch prepared the first drafts of the paper. Stig Lenhoff read and made substantial comments on and contributions to subsequent drafts and approved the final submitted version. All authors read, made comments and approved the final submitted version.

\section{CONFLICT OF INTEREST}

The authors declare no conflict of interest.

\section{REFERENCES}

Andersson I., Ahlberg K., Stockelberg D. \& Persson L.O. (2011) Patients' perception of health-related quality of life during the first year after autologous and allogeneic stem cell transplantation. European Journal of Cancer Care 20, 368-379.
Andrykowski M.A., Brady M.J. \& HensleeDowney P.J. (1994) Psychosocial factors predictive of survival after allogeneic bone marrow transplantation for 
leukemia. Psychosomatic Medicine 56, 432-439.

Andrykowski M.A., Bishop M.M., Hahn E.A., Cella D.F., Beaumont J.L., Brady M.J., Horowitz M.M., Sobocinski K.A., Rizzo J.D. \& Wingard J.R. (2005) Longterm health-related quality of life, growth, and spiritual well-being after hematopoietic stem-cell transplantation. Journal of Clinical Oncology 23, 599-608.

Baumann F.T., Kraut L., Schule K., Bloch W. \& Fauser A.A. (2010) A controlled randomized study examining the effects of exercise therapy on patients undergoing haematopoietic stem cell transplantation. Bone Marrow Transplantation 45, 355-362.

Bevans M.F., Marden S., Leidy N.K., Soeken K., Cusack G., Rivera P., Mayberry H., Bishop M.R., Childs R. \& Barrett A.J. (2006) Health-related quality of life in patients receiving reduced-intensity conditioning allogeneic hematopoietic stem cell transplantation. Bone Marrow Transplantation 38, 101-109.

Bonomi A.E., Cella D.F., Hahn E.A., Bjordal K., Sperner-Unterweger B., Gangeri L., Bergman B., Willems-Groot J., Hanquet P. \& Zittoun R. (1996) Multilingual translation of the Functional Assessment of Cancer Therapy (FACT) quality of life measurement system. Quality of Life Research 5, 309-320.

Bredeson C., Leger C., Couban S., Simpson D., Huebsch L., Walker I., Shore T., Howson-Jan K., Panzarella T., Messner H., Barnett M. \& Lipton J. (2004) An evaluation of the donor experience in the Canadian multicenter randomized trial of bone marrow versus peripheral blood allografting. Biology of Blood and Marrow Transplantation 10, 405-414.

Bush N.E., Donaldson G.W., Haberman M.H., Dacanay R. \& Sullivan K.M. (2000) Conditional and unconditional estimation of multidimensional quality of life after hematopoietic stem cell transplantation: a longitudinal follow-up of 415 patients. Biology of Blood and Marrow Transplantation 6, 576-591.

Carlson L.E., Smith D., Russell J., Fibich C. \& Whittaker T. (2006) Individualized exercise program for the treatment of severe fatigue in patients after allogeneic hematopoietic stem-cell transplant: a pilot study. Bone Marrow Transplantation 37, 945-954.

Cella D.F. (1997) Manual of the Functional Assessment of Chronic Illness Therapy (FACIT) Measurement Systems, 4th edn. Center on Outcomes, Research and Education (CORE), Evanston Northwestern Healthcare and Northwestern University, Evanston, IL, USA.

Cella D.F., Tulsky D.S., Gray G., Sarafian B., Linn E., Bonomi A., Silberman M.,
Yellen S.B., Winicour P. \& Brannon J. (1993) The Functional Assessment of Cancer Therapy scale: development and validation of the general measure. Journal of Clinical Oncology 11, 570579.

Chiodi S., Spinelli S., Ravera G., Petti A.R., Van Lint M.T., Lamparelli T., Gualandi F., Occhini D., Mordini N., Berisso G., Bregante S., Frassoni F. \& Bacigalupo A. (2000) Quality of life in 244 recipients of allogeneic bone marrow transplantation. British Journal of Haematology 110, 614619.

Christopher K.A. (2000) The experience of donating bone marrow to a relative. Oncology Nursing Forum 27, 693-700.

Danaher E.H., Ferrans C., Verlen E., Ravandi F., van Besien K., Gelms J. \& Dieterle N. (2006) Fatigue and physical activity in patients undergoing hematopoietic stem cell transplant. Oncology Nursing Forum 33, 614-624.

DeMarinis V., Barsky A.J., Antin J.H. \& Chang G. (2009) Health psychology and distress after haematopoietic stem cell transplantation. European Journal of Cancer Care 18, 57-63.

Edman L., Larsen J., Hagglund H. \& Gardulf A. (2001) Health-related quality of life, symptom distress and sense of coherence in adult survivors of allogeneic stem-cell transplantation. European Journal of Cancer Care 10, 124-130.

Ferrell B., Grant M., Schmidt G.M., Rhiner M., Whitehead C., Fonbuena P. \& Forman S.J. (1992) The meaning of quality of life for bone marrow transplant survivors. Part 1. The impact of bone marrow transplant on quality of life. Cancer Nursing 15, 153-160.

Fortanier C., Kuentz M., Sutton L., Milpied N., Michalet M., Macquart-Moulin G., Faucher C., Le Corroller A.G., Moatti J.P. \& Blaise D. (2002) Healthy sibling donor anxiety and pain during bone marrow or peripheral blood stem cell harvesting for allogeneic transplantation: results of a randomised study. Bone Marrow Transplantation 29, 145-149.

Gruber U., Fegg M., Buchmann M., Kolb H.J. \& Hiddemann W. (2003) The longterm psychosocial effects of haematopoetic stem cell transplantation. European Journal of Cancer Care 12, 249-256.

Hayes S., Davies P.S., Parker T., Bashford J. \& Newman B. (2004) Quality of life changes following peripheral blood stem cell transplantation and participation in a mixed-type, moderate-intensity, exercise program. Bone Marrow Transplantation 33, 553-558.

Heinonen H., Volin L., Uutela A., Zevon M., Barrick C. \& Ruutu T. (2001a) Quality of life and factors related to perceived satisfaction with quality of life after allogeneic bone marrow transplantation. Annals of Hematology 80, 137-143.

Heinonen H., Volin L., Uutela A., Zevon M., Barrick C. \& Ruutu T. (2001b) Gender-associated differences in the quality of life after allogeneic BMT. Bone Marrow Transplantation 28, 503-509.

Hoodin F., Kalbfleisch K.R., Thornton J. \& Ratanatharathorn V. (2004) Psychosocial influences on 305 adults' survival after bone marrow transplantation; depression, smoking, and behavioral selfregulation. Journal of Psychosomatic Research 57, 145-154.

Kisch A., Dykes J., Lindmark A. \& Lenhoff S. (2008) A proposed plan for the management of adult sibling donors. Bone Marrow Transplantation 42, 357-358.

Kopp M., Schweigkofler H., Holzner B., Nachbaur D., Niederwieser D., Fleischhacker W.W., Kemmler G. \& SpernerUnterweger B. (2000) EORTC QLQ-C30 and FACT-BMT for the measurement of quality of life in bone marrow transplant recipients: a comparison. European Journal of Haematology 65, 97-103.

Larsen J., Nordstrom G., Ljungman P. \& Gardulf A. (2007) Factors associated with poor general health after stem-cell transplantation. Supportive Care in Cancer 15, 849-857.

Lee S.J., Kim H.T., Ho V.T., Cutler C., Alyea E.P., Soiffer R.J. \& Antin J.H. (2006) Quality of life associated with acute and chronic graft-versus-host disease. Bone Marrow Transplantation 38, 305-310.

McCabe C., Begley C., Collier S. \& McCann S. (2008) Methodological issues related to assessing and measuring quality of life in patients with cancer: implications for patient care. European Journal of Cancer Care 17, 56-64.

McQuellon R.P., Russell G.B., Cella D.F., Craven B.L., Brady M., Bonomi A. \& Hurd D.D. (1997) Quality of life measurement in bone marrow transplantation: development of the Functional Assessment of Cancer Therapy-Bone Marrow Transplant (FACT-BMT) scale. Bone Marrow Transplantation 19, 357-368.

McQuellon R.P., Russell G.B., Rambo T.D., Craven B.L., Radford J., Perry J.J., Cruz J. \& Hurd D.D. (1998) Quality of life and psychological distress of bone marrow transplant recipients: the 'time trajectory' to recovery over the first year. Bone Marrow Transplantation 21, 477-486.

Milne D.J., Mulder L.L., Beelen H.C., Schofield P., Kempen G.I. \& Aranda S. (2006) Patients' self-report and family caregivers' perception of quality of life in patients with advanced cancer: how do they compare? European Journal of Cancer Care 15, 125-132.

Pallua S., Giesinger J., Oberguggenberger A., Kemmler G., Nachbaur D., Clausen J., 
Kopp M., Sperner-Unterweger B. \& Holzner B. (2010) Impact of GvHD on quality of life in long-term survivors of haematopoietic transplantation. Bone Marrow Transplantation 45, 1534-1539.

Parker P.A., Baile W.F., de Moor C. \& Cohen L. (2003) Psychosocial and demographic predictors of quality of life in a large sample of cancer patients. PsychoOncology 12, 183-193.

Peterman A.H., Fitchett G., Brady M.J., Hernandez L. \& Cella D. (2002) Measuring spiritual well-being in people with cancer: the functional assessment of chronic illness therapy - Spiritual Wellbeing Scale (FACIT-Sp). Annals of Behavioral Medicine 24, 49-58.

Pidala J., Anasetti C. \& Jim H. (2009) Quality of life after allogeneic hematopoietic cell transplantation. Blood 114, 7-19.

Pidala J., Anasetti C. \& Jim H. (2010) Health-related quality of life following haematopoietic cell transplantation: patient education, evaluation and intervention. British Journal of Haematology 148, 373-385.

Prieto J.M., Saez R., Carreras E., Atala J., Sierra J., Rovira M., Batlle M., Blanch J., Escobar R., Vieta E., Gomez E., Rozman C. \& Cirera E. (1996) Physical and psychosocial functioning of 117 survivors of bone marrow transplantation. Bone Marrow Transplantation 17, 1133-1142.
Schulz-Kindermann F., Mehnert A., Scherwath A., Schirmer L., Schleimer B., Zander A.R. \& Koch U. (2007) Cognitive function in the acute course of allogeneic hematopoietic stem cell transplantation for hematological malignancies. Bone Marrow Transplantation 39, 789-799.

Syrjala K.L., Chapko M.K., Vitaliano P.P., Cummings C. \& Sullivan K.M. (1993) Recovery after allogeneic marrow transplantation: prospective study of predictors of long-term physical and psychosocial functioning. Bone Marrow Transplantation 11, 319-327.

Takita M., Tanaka Y., Kodama Y., Murashige N., Hatanaka N., Kishi Y., Matsumura T., Ohsawa Y. \& Kami M. (2011) Data mining of mental health issues of non-bone marrow donor siblings. Journal of Clinical Bioinformatics 1, 1-7.

van Walraven S.M., Nicoloso-de Faveri G., Axdorph-Nygell U.A., Douglas K.W. Jones D.A., Lee S.J., Pulsipher M., Ritchie L., Halter J., Shaw B.E. \& WMDA Ethics and Clinical working groups (2010) Family donor care management: principles and recommendations. Bone Marrow Transplantation 45, 1269-1273.

van Weert E., Hoekstra-Weebers J., Otter R., Postema K., Sanderman R. \& van der Schans C. (2006) Cancer-related fatigue: predictors and effects of rehabilitation. The Oncologist 11, 184-196.
WHOQOL Group (1993) Study protocol for the World Health Organization project to develop a quality of life assessment instrument. Quality of Life Research 2, 153-159.

Wiener L.S., Steffen-Smith E., Battles H.B., Wayne A., Love C.P. \& Fry T. (2008) Sibling stem cell donor experiences at a single institution. Psycho-Oncology 17, 304-307.

Williams S., Green R., Morrison A., Watson D. \& Buchanan S. (2003) The psychosocial aspects of donating blood stem cells: the sibling donor perspective. Journal of Clinical Apheresis 18, 1-9.

Wilson R.W., Jacobsen P.B. \& Fields K.K. (2005) Pilot study of a home-based aerobic exercise program for sedentary cancer survivors treated with hematopoietic stem cell transplantation. Bone Marrow Transplantation 35, 721-727.

Winterling J., Sidenvall B., Glimelius B. \& Nordin K. (2009) Expectations for the recovery period after cancer treatment - a qualitative study. European Journal of Cancer Care 18, 585-593.

Wong F.L., Francisco L., Togawa K., Bosworth A., Gonzales M., Hanby C., Sabado M., Grant M., Forman S.J. \& Bhatia S. (2010) Long-term recovery after hematopoietic cell transplantation: predictors of quality-of-life concerns. Blood 115, 25082519. 